\title{
Transcutaneous intravascular transposition of a permanent dialysis catheter
}

\author{
Jerzy Piecuch ${ }^{1}$, Maciej Wiewióra ${ }^{1}$, Wojciech Jacheć ${ }^{2}$, Monika Szrot ${ }^{1}$, Albert Krzak ${ }^{3}$ \\ ${ }^{1}$ Department of General and Bariatric Surgery and Emergency Medicine in Zabrze, Medical University of Silesia, Katowice, Poland \\ $2^{2} 2^{\text {nd }}$ Department of Cardiology in Zabrze, Medical University of Silesia, Katowice, Poland \\ ${ }^{3}$ Student Scientific Society at Department of General and Bariatric Surgery and Emergency Medicine in Zabrze, Medical University \\ of Silesia, Katowice, Poland
}

Videosurgery Miniin (e-pub, ahead of print) DOI: $10.5114 /$ wiitm.2014.44136

\begin{abstract}
Permanent catheters are used to provide long-lasting access for long-term dialysis therapy in certain patients when creating an arteriovenous fistula between their own vessels is not possible, when there are contraindications for fistula creation or when the expected lifetime of the patient is short. We present the case of a patient with terminal renal insufficiency treated with hemodialysis for 5 years. Due to post-thrombotic changes and venous stenosis resulting from long-term cannulation with dialysis catheters, the replacement catheter was inserted through the previously used canal and transcutaneous intravascular catheter transposition was performed.
\end{abstract}

Key words: permanent catheters, hemodialysis, endovascular catheter transposition.

\section{Introduction}

Renal replacement therapy in terminal renal insufficiency requires appropriate vascular access. An arteriovenous fistula made from the patient's own vessels is the best form of access for hemodialysis $[1,2]$. When creating a fistula is not possible, dialysis catheters are most commonly used to provide temporary access. In some unique cases, when creating a fistula between a patient's own vessels is not possible, when there are contraindications for fistula creation or the expected lifetime of a patient is short, a permanent catheter is used to provide longterm access [3].

We present the case of a patient with terminal renal insufficiency in which a transcutaneous intravascular permanent catheter transposition was performed for dialysis.

\section{Case report}

The patient was 83-years-old and presented with chronic renal failure that had been treated by hemodialysis for 5 years. The patient was admitted to the hospital due to the occlusion of a Demers catheter that was implanted a year previously in the left internal jugular vein. Previously, she had required several alterations in her vascular access due to catheter occlusion or cannulated vein thrombosis. The patient also suffered from arterial hypertension and coronary disease after a myocardial infarction. Due to the post-thrombotic changes and venous stenosis as a result of the long-term cannulation with dialysis catheters, the subsequent catheter replacement was performed through the canal used previously for the implantation of the Demers catheter. In the operating theater, a tunneled portion of the catheter

\section{Address for correspondence}

Maciej Wiewióra MD, PhD, Department of General and Bariatric Surgery and Emergency Medicine, Medical University of Silesia,

10 Sklodowskiej-Curie St, 41-800 Zabrze, Poland, phone: +48 3237323 81, e-mail: m-wiewiora@tlen.pl 

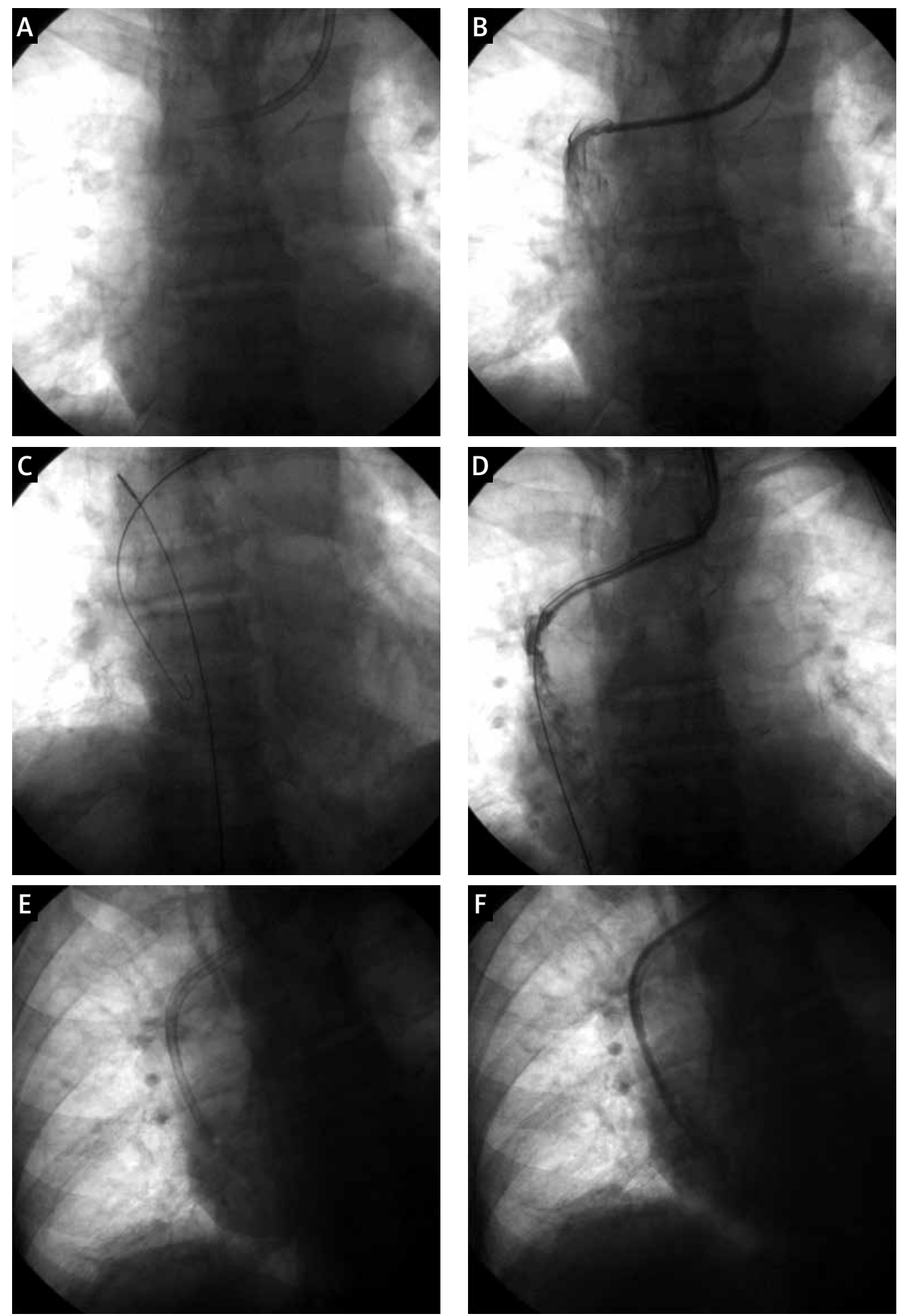

Photo 1. All stages of dialysis catheter transposition (for a more detailed description, see the text): $\mathbf{A}-$ The port end was positioned in the distal part of the obstructed left brachiocephalic vein. $\mathbf{B}$ - Confirmation of the port patency. C - Introduction of the "J" guide wire into the right atrium and the loop from the right femoral vein access. D, E - Port transposition into the right atrium. F - Confirmation of the patency of the dialysis catheter 
was dissected free. The catheter was shortened and the guide wire used for inserting the new permanent catheter was then introduced through the proximal end of the truncated catheter. The X-ray examination confirmed the correct positioning of the catheter after implantation. The patient, now with a correctly functioning catheter, was moved directly to the dialysis ward. The next day the patient returned from the dialysis ward because effective dialysis was not possible. Repositioning the catheter on the implantation side was not possible. Venography through the dialysis catheter showed that the port end was dislocated to the distal part of the post-thrombotic obstructed right brachiocephalic vein (Photo 1 A). There was a visible $2-\mathrm{cm}$ canal length and a narrow channel of the diameter of the port connecting with the superior vena cava. Flow through the catheter was retained in both canals (Photo $1 \mathrm{~B}$ ). Aspiration was possible due to a valve mechanism. An attempt to lower the catheter end into the right atrium was unsuccessful, even after stiffening the port with a stiff guide wire. A "J" 0.035 " guide wire was implanted in the right atrium through the central canal. The introducer was inserted into the right femoral vein using the Seldinger technique and the guide wire was then secured with a 30-mm loop held with Pean forceps to prevent aspiration into the port (Photo $1 \mathrm{C}$ ). Using this traction while simultaneously pushing the port, transposition into the right atrium lumen was achieved (Photos $1 \mathrm{D}$ and $1 \mathrm{E}$ ). As a result, flow through the catheter in both directions was restored (Photo $1 \mathrm{~F}$ ).

\section{Discussion}

Currently, both acute and permanent catheters are used for hemodialysis. Temporary catheters are used in situations in which immediate hemodialysis treatment is required and the catheters in the central vein will not be necessary for longer than several weeks. If the predicted time of dialysis therapy is longer, if venous access is not possible through a dialysis fistula, or if there are other contraindications for temporary catheterization, permanent catheters are utilized [4]. Their presence is connected with both early and late complications. The most common complications include bleeding, infection, catheter thrombosis and cannulation of the vena cava [5]. Maintaining well-functioning vascular access in patients with terminal renal insufficiency is a prior- ity. In cases of catheter occlusion or arteriovenous fistula, a variety of methods are used including local fibrinolysis and intravascular recanalization $[6,7]$. Catheter thrombosis usually requires catheter replacement if other methods of restoring its patency are ineffective. Catheter replacement often requires a new vascular access, which can be very difficult in cases of long-term dialysis. This situation occurred in the presented patient. Subsequent catheter replacement was accomplished through the canal of the previously implanted Demers catheter. This catheter insertion route was used because the imaging examinations revealed that this was the only possible site for implantation of the dialysis catheter.

\section{Conclusions}

Catheter replacement using a previous access route in connection with intravascular techniques is an alternative method for obtaining vascular access for dialysis.

\section{References}

1. Kumano S, Itatani K, Shiota J, et al. Strategies for the creation and maintenance of reconstructed arteriovenous fistulas using the forearm basilic vein. Ther Apher Dial 2013; 17: 504-9.

2. Praga M, Merello J, Palomares I, et al. Type of vascular access and survival among very elderly hemodialysis patients. Nephron Clin Pract 2013; 124: 47-53.

3. Leś J, Wańkowicz Z. Methods of central vascular access for haemodialysis. Anaesthesiol Intensive Ther 2013; 45: 171-6.

4. Cetinkaya R, Odabas AR, Unlu Y, et al. Using cuffed and tunnelled central venous catheters as permanent vascular access for hemodialysis: a prospective study. Ren Fail 2003; 25: 431-8.

5. Ravani P, Gillespie BW, Quinn RR, et al. Temporal risk profile for infectious and noninfectious complications of hemodialysis access. J Am Soc Nephrol 2013; 24: 1668-77.

6. Mendes ML, Castro JH, Silva TN, et al. Effective use of alteplase for occluded tunneled venous catheter in hemodialysis patients. Artif Organs 2014; 38: 399-403.

7. Kamper L, Piroth W, Haage P, et al. Endovascular treatment of dysfunctional hemodialysis catheters. J Vasc Access 2010; 11: 263-8.

Received: 18.12.2013, accepted: 5.03.2014. 